Review began 01/27/2022 Review ended 02/11/2022 Published 02/20/2022

() Copyright 2022

Kumar et al. This is an open access article distributed under the terms of the Creative Commons Attribution License CC-BY 4.0. which permits unrestricted use, distribution, and reproduction in any medium, provided the original author and source are credited.

\section{A Comparative Analysis of COVID-19 IgG Antibody Level and Socio-Demographic Status in Symptomatic and Asymptomatic Population of South Andaman, India}

\author{
Deepak Kumar ${ }^{1}$, Amrita Burma ${ }^{1}$, Ashish Kumar Mandal ${ }^{2}$, Vidhu Joshy ${ }^{3}$ \\ 1. Department of Community Medicine, Andaman and Nicobar Islands Institute of Medical Sciences, Port Blair, IND 2 \\ Department of Pathology, Andaman and Nicobar Islands Institute of Medical Sciences, Port Blair, IND 3. Department \\ of Biostatistics, Andaman and Nicobar Islands Institute of Medical Sciences, Port Blair, IND
}

Corresponding author: Ashish Kumar Mandal, equanimity1410@gmail.com

\begin{abstract}
Introduction: The serosurveillance of COVID-19 antibody levels and their difference between symptomatic and asymptomatic groups can help in understanding the immune status of the community and the factors affecting it. Hence, the study was undertaken to find the differences between these two groups with respect to antibodies level and other socio-demographic variables in the South Andaman district.
\end{abstract}

Methods: A population-based serosurveillance study covering more than 4,000 samples was carried out in the South Andaman district. The participants were selected by multistage cluster sampling. The venous blood samples were tested for IgG COVID-19 antibodies by Erba Lisa Elisa kit.

Results: $5.3 \%$ of total individuals (217) were symptomatic whereas $94.7 \%(3,872)$ were asymptomatic. The symptomatic individuals had lower antibodies (33.6\%) as compared to asymptomatic individuals (40.1\%) (pvalue $=0.059$ ). In the age group of 31-45 years, antibody positivity in the asymptomatic group was significantly higher than in the symptomatic group (p-value 0.031 ). The antibody positivity was higher in moderate to severe cases who needed hospital admission. The antibody positivity was found similar in both the groups in front-line workers as well as in non-front-line workers ( $p$-value $=0.104,0.274$, respectively).

Conclusion: The antibody positivity was higher in asymptomatic individuals as compared to symptomatic individuals, particularly in the age group of 31-45 years. The higher level of antibody positivity in asymptomatic individuals reflected a stronger immune response which led to no clinical manifestations. The antibody positivity was also found higher in moderate to severe cases undergoing hospital admission whereas antibodies positivity was found similar in front-line and non-front-line workers.

Categories: Infectious Disease, Epidemiology/Public Health

Keywords: sero-survey, symptomatic infection, covid 19, immunity, asymptomatic infection, andaman

\title{
Introduction
}

Coronavirus disease (COVID-19) emerged in December 2019 and spread across the world since then [1,2]. Till February 14, 2021 over 108.2 million cases and 2.38 million deaths have been reported globally [3]. Hence, a need was felt to conduct a serosurveillance of the COVID-19 antibody among the population for understanding the immune status and the factors that affect it. This may also help policy-makers in formulating better preventive strategies for the prevention of COVID-19 in communities.

International studies from Sweden and UAE have observed the association of seroprevalence of COVID-19 antibodies with self-reports symptoms $[4,5]$. But, the various other seroprevalence studies conducted during the pandemic have not analyzed the differences in antibodies level in symptomatic and asymptomatic individuals [6-8]. Hence, little is known about antibodies difference between these two groups in the world and particularly in an isolated and remote island such as Andaman and Nicobar.

Andaman and Nicobar Islands are remotely located in the Bay of Bengal region and have a segregated and isolated population with its own unique demography. South Andaman district is the most developed district of the whole archipelago and has approximately $60 \%$ of the population of the whole Andaman and Nicobar islands. The whole archipelago is served by only one tertiary care hospital which is located in the South Andaman district resulting in the referral of all the cases of the island to this hospital. Hence, this study was undertaken to find the differences between symptomatic and asymptomatic cases of COVID-19 with respect to antibodies level and other socio-demographic variables in the South Andaman district.

\section{Materials And Methods}


The present study is a cross-sectional study conducted among the population of the South Andaman district. The consenting adults of 18 years and above were included in the study. The individuals suffering from any immune-deficient condition like HIV/or under chemotherapy were excluded from the study. The available literature did not provide any reliable estimates of the prevalence of COVID-19 antibodies in the Andaman and Nicobar Islands. Therefore, the sample size was calculated by considering 50\% prevalence $[9,10]$.

Further, 2.5 design effect, $2.5 \%$ absolute precision were used for sample size calculation. The sample size was calculated by the following formula:

$n=\left[z^{2} p q / d^{2}\right]$ DEFF,

where $\mathrm{n}=$ sample size, $\mathrm{z}=$ linked to $95 \%$ confidence interval for cluster sampling $=2.0, \mathrm{p}=$ expected prevalence $($ fraction of 1$)=0.5, q=1-p$ (expected non-prevalence $)=0.5, d=$ absolute precision $=0.025$, $\mathrm{DEFF}=$ Design Effect $=2.5$. The minimum sample size came out to be 4,000 . Hence, a total of 4,089 individuals were included in the study.

The multistage cluster sampling was used for the selection of participants in the study. A village in the rural area and a municipal ward in urban areas were taken as a cluster for sampling. Based on the unique geography and demography of the island, 45 clusters (27 clusters from urban areas and 18 clusters from rural areas) were selected randomly from the approximate 125 clusters identified in the South Andaman district. In each cluster, 100 participants were selected randomly from the list of eligible residents of the cluster. There was a non-response rate of $9.2 \%$ among the total study population. The list of clusters and permission to conduct the survey was obtained from Andaman and Nicobar administration.

The first wave of COVID-19 reached its peak in August 2020 in Andaman and Nicobar Islands and it started to decline after one month [11]. Hence, the sample collection was done from December 15, 2020 to February 14,2021 to find the status of immunity in symptomatic and asymptomatic individuals after the first wave of COVID-19 pandemic exposure on the island. The survey team collected information about the sociodemographic profiles (age, sex, geography, occupation) and medical/clinical profiles of the participants by a predesigned and pre-validated questionnaire. The survey team collected 3-5 mL venous blood samples for detecting COVID-19 antibodies. The procedure of data and blood collection was done only after the informed consent of the participants.

The health care workers were included in front-line workers and the rest of the participants were included in non-front line workers. The symptoms of participants that occurred in the past one month of survey were categorized according to guidelines issued by the All India Institute of Medical Science (AIIMS)/Indian Council of Medical Research (ICMR) COVID-19 national task force [12].

COVID-19 IgG antibodies were detected by using Erba Lisa ELISA-based test. This kit was based on the principle of indirect ELISA using recombinant Spike subunit antigen. The antibody positivity was found by calculating the antibody index. The antibody index was calculated by dividing each sample's optical density (OD) by cut-off values. The antibody index with less than 0.9 was reported as non-detectable IgG antibody for COVID-19 whereas an antibody index greater than 0.9 was reported as a detectable IgG antibody for COVID-19.

SPSS version 20.0 (IBM, Armonk, NY, USA) was used for data analysis. Descriptive analysis was done for socio-demographic variables like age, sex, residence, and occupation. The association of various categorical variables (like age, sex, residence, occupation, COVID-19 contact, hospital admission, medical consultation, previous test for COVID-19) with antibody positivity in symptomatic and asymptomatic individuals were compared with Chi-square/Fisher Exact test. The OD values of symptoms related to COVID-19 infection were analyzed with the Mann-Whitney U test. A p-value of $<0.05$ was considered statistically significant. The study was approved by the Institutional ethical committee of ANIIMS, Port Blair.

\section{Results}

Among the population residing in South Andaman Islands, 4,089 persons were examined for COVID-19 antibodies. Out of this, $19.3 \%$ belonged to $18-30$ years, $43.2 \%$ belonged to $31-45$ years and $37.5 \%$ were above 46 years. According to gender-wise distribution, $57.0 \%$ of the population were females whereas $43.0 \%$ were males. The proportion of participants residing in urban areas was $62.9 \%$ whereas the proportion of participants residing in rural areas was $37.1 \%$.

The symptoms of COVID-19 infections post the first wave of the COVID-19 pandemic in Andaman were present in 217 (5.3\% of total) individuals whereas 3872 (94.7\% of total) individuals were asymptomatic. The antibody positivity of the study individuals was reflected by the antibody index. The percentage of symptomatic people (33.6\%) who were positive for antibodies was lower than asymptomatic individuals (40.1\%) (Table 1). 


\section{Cureus}

\begin{tabular}{|c|c|c|c|c|c|c|}
\hline & \multicolumn{4}{|c|}{ COVID-19 Antibody (Ab index) } & \multirow{3}{*}{ Total } & \multirow{3}{*}{ P-value (chi-square test) } \\
\hline & \multicolumn{2}{|c|}{ Positive } & \multicolumn{2}{|c|}{ Negative } & & \\
\hline & $\mathrm{N}$ & $\%$ & $\mathrm{n}$ & $\%$ & & \\
\hline Symptomatic & 73 & $33.6 \%$ & 144 & $66.4 \%$ & 217 & \multirow{3}{*}{0.059} \\
\hline Asymptomatic & 1,552 & $40.1 \%$ & 2,320 & $59.9 \%$ & 3,872 & \\
\hline Total & 1,625 & $39.7 \%$ & 2,464 & $60.3 \%$ & 4,089 & \\
\hline
\end{tabular}

TABLE 1: The comparison of COVID-19 antibody positivity in symptomatic and asymptomatic individuals in the South Andaman Islands.

The association of various socio-demographic variables like age, sex, geography, occupation with antibody positivity in symptomatic and asymptomatic individuals was analyzed as shown in Table 2 . The antibody positivity in the symptomatic group was $28.0 \%$ as compared to $34.0 \%$ in the asymptomatic group in age groups of 18-30 years. Similarly in the age group of 31-45 years, asymptomatic individuals had significantly higher antibodies $(40.9 \%)$ as compared to symptomatic individuals $(29.9 \%)$ ( $\mathrm{p}$-value $=0.031)$. The gender-wise analysis of antibody positivity in symptomatic and asymptomatic groups revealed that there was no significant difference in antibody positivity in symptomatic males (32.1\%) as compared to asymptomatic males (38.1\%) ( $\mathrm{p}$-value $=0.269)$. Similarly, it was not statistically different ( $\mathrm{p}$-value $=0.111)$ in symptomatic females (34.6\%) as compared to asymptomatic females (41.6\%).

The antibody positivity in asymptomatic rural individuals (41.1\%) was also similar to symptomatic rural individuals (27.4\%) ( $\mathrm{p}$-value $=0.097)$. Similarly, the antibody positivity had no difference between symptomatic urban individuals (36.8\%) as compared to asymptomatic urban individuals (41.9\%) (pvalue $=0.225$ ). The comparisons of antibody positivity in symptomatic individuals and asymptomatic individuals in relation to front-line workers did not show any significant difference ( $\mathrm{p}$-value $=0.104$ ). Similarly, the antibody positivity was also found similar ( $p$-value=0.274) between symptomatic non-frontline workers (34.5\%) and asymptomatic non-front-line workers (39.6\%) (Table 2). 


\section{Cureus}

\begin{tabular}{|c|c|c|c|c|c|c|c|}
\hline \multirow{3}{*}{ Socio-demographic Variable } & \multirow{3}{*}{ Symptoms } & \multicolumn{4}{|c|}{ COVID-19 Antibody } & \multirow{3}{*}{ Total } & \multirow{3}{*}{ P-value } \\
\hline & & \multicolumn{2}{|c|}{ Positive } & \multicolumn{2}{|c|}{ Negative } & & \\
\hline & & $\mathrm{n}$ & $\%$ & $\mathrm{~N}$ & $\%$ & & \\
\hline \multirow{2}{*}{ Age (18-30years) } & Symptomatic & 14 & $28.6 \%$ & 35 & $71.4 \%$ & 49 & \multirow{2}{*}{0.435} \\
\hline & Asymptomatic & 252 & $34.0 \%$ & 489 & $66.0 \%$ & 741 & \\
\hline \multirow{2}{*}{ Age (31-45years) } & Symptomatic & 29 & $29.9 \%$ & 68 & $70.1 \%$ & 97 & \multirow{2}{*}{0.031} \\
\hline & Asymptomatic & 683 & $40.9 \%$ & 987 & $59.1 \%$ & 1,670 & \\
\hline \multirow{2}{*}{ Age ( $\geq 46 y e a r s)$} & Symptomatic & 30 & $42.3 \%$ & 41 & $57.7 \%$ & 71 & \multirow{2}{*}{0.997} \\
\hline & Asymptomatic & 617 & $42.2 \%$ & 844 & $57.8 \%$ & 1,461 & \\
\hline \multirow{2}{*}{ Male } & Symptomatic & 27 & $32.1 \%$ & 57 & $67.9 \%$ & 84 & \multirow{2}{*}{0.269} \\
\hline & Asymptomatic & 639 & $38.1 \%$ & 1,037 & $61.9 \%$ & 1,676 & \\
\hline \multirow{2}{*}{ Female } & Symptomatic & 46 & $34.6 \%$ & 87 & $65.4 \%$ & 133 & \multirow{2}{*}{0.111} \\
\hline & Asymptomatic & 913 & $41.6 \%$ & 1,283 & $58.4 \%$ & 2,196 & \\
\hline \multirow{2}{*}{ Rural } & Symptomatic & 20 & $27.4 \%$ & 53 & $72.6 \%$ & 73 & \multirow{2}{*}{0.097} \\
\hline & Asymptomatic & 533 & $37.0 \%$ & 909 & $63.0 \%$ & 1,442 & \\
\hline \multirow{2}{*}{ Urban } & Symptomatic & 53 & $36.8 \%$ & 91 & $63.2 \%$ & 144 & \multirow{2}{*}{0.225} \\
\hline & Asymptomatic & 1019 & $41.9 \%$ & 1,411 & $58.1 \%$ & 2,430 & \\
\hline \multirow{2}{*}{ Front-line workers } & Symptomatic & 33 & $32.7 \%$ & 68 & $67.3 \%$ & 101 & \multirow{2}{*}{0.104} \\
\hline & Asymptomatic & 644 & $40.8 \%$ & 933 & $59.2 \%$ & 1,577 & \\
\hline \multirow{2}{*}{ Non-front line workers } & Symptomatic & 40 & $34.5 \%$ & 76 & $65.5 \%$ & 116 & \multirow{2}{*}{0.274} \\
\hline & Asymptomatic & 908 & $39.6 \%$ & 1,387 & $60.4 \%$ & 2,295 & \\
\hline
\end{tabular}

TABLE 2: The association of various socio-demographic variables and COVID-19 antibodies among symptomatic and asymptomatic individuals.

Out of the study participants, only 5.3\% ( $\mathrm{n}=217$ ) individuals had COVID-19 symptoms. The fever was the most common symptom (41\%) followed by cough (35.9\%) and cold (26.7\%). Most of the symptoms such as fever (41\%), cough (35.9\%), cold (26.7\%), sore throat (8.7\%), myalgia (5.1\%), headache (4.6\%), and loss of taste $(0.9 \%)$ were milder. The moderate/ severe symptoms like dyspnoea/breathing difficulty were observed only in $1.8 \%(n=4)$ of total symptomatic patients ( $\mathrm{n}=217)$. The COVID- 19 antibody level (OD value) was similar irrespective of the type of symptoms (Table 3). 


\section{Cureus}

\begin{tabular}{|c|c|c|c|c|c|}
\hline \multirow{2}{*}{ History of symptoms } & \multicolumn{4}{|c|}{ OD value } & \multirow{2}{*}{ P-value (Mann-Whitney U test) } \\
\hline & Mean & Std. Deviation & Minimum & Maximum & \\
\hline Fever & 0.229 & 0.308 & 0 & 1.288 & \multirow{2}{*}{0.915} \\
\hline No fever & 0.253 & 0.405 & 0 & 2.262 & \\
\hline Cough & 0.271 & 0.437 & 0 & 2.262 & \multirow{2}{*}{0.211} \\
\hline No cough & 0.227 & 0.323 & 0 & 1.872 & \\
\hline Sore throat & 0.179 & 0.234 & 0 & 0.852 & \multirow{2}{*}{0.374} \\
\hline No sore throat & 0.249 & 0.378 & 0 & 2.262 & \\
\hline Cold & 0.257 & 0.456 & 0 & 2,262 & \multirow{2}{*}{0.669} \\
\hline No cold & 0.237 & 0.332 & 0 & 1.872 & \\
\hline Headache & 0.331 & 0.567 & 0 & 1.872 & \multirow{2}{*}{0.796} \\
\hline No headache & 0.238 & 0.357 & 0 & 2.262 & \\
\hline Dyspnoea & 0.246 & 0.370 & 0 & 2.262 & \multirow{2}{*}{0.632} \\
\hline No Dyspnoea & 0.224 & 0.375 & 0 & 1.872 & \\
\hline Myalgia & 0.131 & 0.119 & 0.007 & 0.372 & \multirow{2}{*}{0.964} \\
\hline No myalgia & 0.248 & 0.376 & 0 & 2.262 & \\
\hline Loss of taste & 0.094 & 0.096 & 0.026 & 0.163 & \multirow{2}{*}{0.838} \\
\hline No loss of taste & 0.244 & 0.369 & 0.007 & 0.372 & \\
\hline
\end{tabular}

TABLE 3: The association of symptoms with COVID-19 antibody (OD value).

OD - optical density

The percentage positivity of antibodies in symptomatic and asymptomatic individuals with a history of contact was compared and it was found to be similar (Table 4). The seropositivity in symptomatic (32.8\%) and asymptomatic individuals (40.5\%) who were never tested before for COVID-19 by RT-PCR had no statistically significant difference ( $\mathrm{p}$-value $=0.075$ ). Similarly, the seropositivity was also compared between symptomatic (35.0\%) and asymptomatic (39.1\%) individuals who were previously tested at least once by RTPCR, and it was found to be similar ( $\mathrm{p}$-value $=0.470$ ). The RT-PCR tested group was further divided into two subgroups, negative and positive for COVID-19. Reviewer beta: groups. In both these subgroups, the symptomatic and asymptomatic individuals had similar percentage positivity (Table 4). 


\section{Cureus}

\begin{tabular}{|c|c|c|c|c|c|c|c|}
\hline \multirow{3}{*}{ Medical History } & \multirow{3}{*}{ Symptoms } & \multicolumn{4}{|c|}{ COVID-19 Antibody } & \multirow{3}{*}{ Total } & \multirow{3}{*}{ P-value } \\
\hline & & \multicolumn{2}{|c|}{ Positive } & \multicolumn{2}{|c|}{ Negative } & & \\
\hline & & $\mathrm{n}$ & $\%$ & $\mathrm{~N}$ & $\%$ & & \\
\hline \multirow{2}{*}{ No history of COVID-19 contacts } & Symptomatic & 59 & $33.3 \%$ & 118 & $66.7 \%$ & 177 & \multirow{2}{*}{0.084} \\
\hline & Asymptomatic & 1,455 & $39.8 \%$ & 2,198 & $60.2 \%$ & 3,653 & \\
\hline \multirow{2}{*}{ History of COVID-19 contacts } & Symptomatic & 14 & $35.0 \%$ & 26 & $65.0 \%$ & 40 & \multirow{2}{*}{0.274} \\
\hline & Asymptomatic & 97 & $44.3 \%$ & 122 & $55.7 \%$ & 219 & \\
\hline \multirow{2}{*}{ Previously never tested for COV } & Symptomatic & 45 & $32.8 \%$ & 92 & $67.2 \%$ & 137 & \multirow{2}{*}{0.075} \\
\hline & Asymptomatic & 1,123 & $40.5 \%$ & 1,651 & $59.5 \%$ & 2,774 & \\
\hline \multirow{2}{*}{ Previously at least once tested for COVID-19 by RT-PCR } & Symptomatic & 28 & $35.0 \%$ & 52 & $65.0 \%$ & 80 & \multirow{2}{*}{0.470} \\
\hline & Asymptomatic & 429 & $39.1 \%$ & 669 & $60.9 \%$ & 1,098 & \\
\hline \multirow{2}{*}{ Previously tested negative COVID-19 by RT-PCR } & Symptomatic & 19 & $28.8 \%$ & 47 & $71.2 \%$ & 66 & \multirow{2}{*}{0.430} \\
\hline & Asymptomatic & 315 & $33.5 \%$ & 625 & $66.5 \%$ & 940 & \\
\hline \multirow{2}{*}{ Previously tested positive COVID-19 by RT-PCR } & Symptomatic & 9 & $64.3 \%$ & 5 & $35.7 \%$ & 14 & \multirow{2}{*}{0.531} \\
\hline & Asymptomatic & 114 & $72.2 \%$ & 44 & $27.8 \%$ & 158 & \\
\hline
\end{tabular}

TABLE 4: The association of clinical history with COVID-19 antibodies in symptomatic and asymptomatic individuals.

To correlate the antibody level with the severity of the symptoms, antibody levels were correlated with hospital admission since only moderate to severely symptomatic cases were admitted to the hospital. The symptomatic individuals who were admitted to the hospital had higher COVID-19 antibodies (61.9\%) as compared to symptomatic individuals who were not admitted to the hospital (30.6\%), (p-value 0.003$)$. However, when the treatment and medical consultation were compared between symptomatic and asymptomatic individuals, the antibody levels were found to be similar (Table 5).

\begin{tabular}{|c|c|c|c|c|c|c|}
\hline & \multicolumn{4}{|c|}{ COVID-19 Antibody } & \multirow{3}{*}{ Total } & \multirow{3}{*}{ P-value (chi-square test) } \\
\hline & \multicolumn{2}{|c|}{ Positive } & \multicolumn{2}{|c|}{ Negative } & & \\
\hline & $\mathrm{N}$ & $\%$ & $\mathrm{~N}$ & $\%$ & & \\
\hline No medical consultation & 46 & $37.4 \%$ & 77 & $62.6 \%$ & 123 & \multirow{2}{*}{0.180} \\
\hline Medical consultation done & 27 & $28.7 \%$ & 67 & $71.3 \%$ & 94 & \\
\hline No Hospital admission & 60 & $30.6 \%$ & 136 & $69.4 \%$ & 196 & \multirow{2}{*}{0.003} \\
\hline Admitted in Hospital & 13 & $61.9 \%$ & 8 & 38.1 & 21 & \\
\hline
\end{tabular}

TABLE 5: The association of treatment history with COVID-19 antibodies in symptomatic individuals.

\section{Discussion}

The present study was conducted among the population of remote South Andaman Island to find the immune status of the community and the factors affecting it, whether a difference existed between the symptomatic and asymptomatic manifestation of the infection. The serosurveillance revealed that $94.7 \%$ of the individuals were asymptomatic. Similar incidences ranging from $90 \%$ to $98 \%$ were reported in the survey conducted in Delhi, Orissa, and the second round of the National Survey of ICMR [13-15]. These studies reported a higher incidence of asymptomatic individuals in respective study areas which happened due to 
widespread infection and asymptomatic seroconversion in the respective communities. On the contrary, the asymptomatic infections in many Asians and European countries ranged from $18 \%$ to $81 \%$, which might have happened due to the variable density of population in these countries and variation of containment measures [16].

When the antibody level was compared between symptomatic and asymptomatic groups then it revealed that antibody positivity was higher in asymptomatic individuals (40.1\%) as compared to symptomatic individuals. The higher level of antibody positivity in asymptomatic individuals reflected a stronger immune response which subdued the infection to such a level that the case did not manifest at all [17,18]. It was further observed that in the age group of 31 to 45 years significantly higher percentage of asymptomatic individuals were antibody positive compared to the symptomatic group, probably being the working-age group they had a higher incidence of exposure. Their positivity was also higher than older individuals. This may be because at a younger age they are likely to have a more effective immune response compared to older people [19]. Further older people with comorbidities would always have decreased immunity compared to younger people [20].

In the cases that were previously RT-PCR positive compared to those who became positive later, the people who later became positive, antibody positivity was higher as compared to people who were infected previously as the time gap between these two groups was approximately $2-3$ months. Hence, it may be assumed that by the time antibody was tested in the previously positive group, the antibody level had started coming down. Thus, it was lower than a recently infected group. Many studies also revealed slowly fading antibodies over a period of 14 days to 4 months in asymptomatic and mild manifestations whereas antibodies persisted comparatively for a longer duration in severe cases as an immune response was strong in them [20-24]. Further, there was no significant antibody difference between the groups which were never tested or tested at least once because majority cases suffered from asymptomatic infection and the positivity rate of RT-PCR is around $80 \%[14,15]$.

Antibody positivity was also found similar in symptomatic and asymptomatic individuals who had contact with COVID-19 positive cases as compared to symptomatic and asymptomatic individuals who had no contact with COVID-19 positive cases. The present study was done after the first pandemic but before starting of the second COVID-19 pandemic. Hence, the COVID-19 exposure and subsequent antibody development occurred due to exposure during the first wave. During this wave and a subsequent one to two months, the lockdown was very severe and strictly implemented. Public transport was completely shut down. Quarantine was strictly followed. Infected people and their contacts were kept in hotels completely isolated and away from others. Even area wise segregation and isolation were enforced. The areas were divided into three zones. The highest infectivity zone had the severest lockdown followed by the moderate infectivity zone and the rest of the areas were COVID-19 free zone. People were confined to their homes in a severe and moderate infective zone with restrictions of movement. The facemask and sanitization were strictly followed a violation was penalized. In spite of this people became seropositive across all the zones and developed a similar level of the antibody as is seen in this study. Hence the virus spread defying all the barriers. So, other than spreading through touch or close contacts there appeared to be an alternate mechanism of transmission and spread. It is known that aerosol spread can effectively bypass these mechanical barriers as they remain suspended in air over long distances and time. WHO has also suggested that aerosol is another mode of spread of this virus [25,26]. This probably was the reason that the demographic variables like gender, urban and rural divide and professional profile had no impact on infectivity and hence the antibodies level or clinical manifestations.

Similarly, the frontline workers, as well as non-front-line workers, had similar antibody levels between the symptomatic and asymptomatic group, which was in spite of the fact that their work profiles varied a lot and many did not even come in contact with any patient and they maintained all precautionary measure such as facemask, washing of hands and social distancing. This could only happen if the incidence of infection, irrespective of different factors and environmental conditions, remained similar.

The study also revealed that no single sign and symptom of COVID-19 had any relation with levels of antibodies. In the serosurvey of Orissa, symptoms like breathing difficulty, myalgia, loss of taste/anosmia also had no association with seropositivity [14]. Though there was an association of fever, cough, and diarrhoea with the seropositivity of the participants [14]. This difference might have arisen due to the reason that the symptoms like the fever of longer duration, persistent cough, and diarrhoea are clinical manifestations of severe cases. Our study also found that moderate to severe cases which needed hospital admission had higher antibodies as compared to individuals who did not require hospital admission. Hence, the IgG type of antibody (as detected by our kit) correlated well with the severity of the manifestation. Similarly, another study that severely ill patients that were intubated or passed away due to COVID-19 had the highest levels of IgG and IgA antibodies targeting RBD and spike, but no significant differences were seen for IgM. These individuals also had the highest neutralization titers. In contrast, individuals that were not hospitalized had the lowest IgG and IgA levels and neutralization titers [27]. Few other studies also reported that neutralising antibodies titers correlated strongly with disease severity and with anti-spike IgG levels [28]. The patients from intensive care units exhibited high neutralising antibodies titers; conversely, patients with milder disease symptoms had heterogeneous neutralising antibodies titers, and asymptomatic or exclusive outpatient-care patients had no or low neutralising antibodies [8,20,21]. 


\section{Conclusions}

The current study signifies that viral exposure has led to predominantly asymptomatic infection. The antibody positivity was higher in asymptomatic individuals as compared to symptomatic individuals. The higher level of antibody positivity in asymptomatic individuals reflected a stronger immune response, which led to no clinical manifestations. This was also seen in other age groups, particularly in the working-age group of 31-45 years. The antibody was seen higher in moderate to severe cases. The antibody positivity was found similar in symptomatic and asymptomatic individuals irrespective of other socio-demographic variables like gender, geography, and occupation. The antibody positivity was also found similar in individuals who had contact with COVID-19 positive individuals as compared to individuals who had no contact with COVID-19 positive individuals.

\section{Additional Information Disclosures}

Human subjects: Consent was obtained or waived by all participants in this study. Institutional Ethics Committee ANIIMS Port Blair issued approval F. No. 1/155/ANIIMS/IEC/2021/05. Animal subjects: All authors have confirmed that this study did not involve animal subjects or tissue. Conflicts of interest: In compliance with the ICMJE uniform disclosure form, all authors declare the following: Payment/services info: All authors have declared that no financial support was received from any organization for the submitted work. Financial relationships: All authors have declared that they have no financial relationships at present or within the previous three years with any organizations that might have an interest in the submitted work. Other relationships: All authors have declared that there are no other relationships or activities that could appear to have influenced the submitted work.

\section{Acknowledgements}

We would like to thank Andaman and Nicobar administration for their necessary help and support.

\section{References}

1. Munster VJ, Koopmans M, van Doremalen N, van Riel D, de Wit E: A novel coronavirus emerging in Chinakey questions for impact assessment. N Engl J Med. 2020, 382:692-4. 10.1056/NEJMp2000929

2. Li Q, Guan X, Wu P, et al.: Early transmission dynamics in Wuhan, China, of novel coronavirus-infected pneumonia. N Engl J Med. 2020, 382:1199-207. 10.1056/NEJMoa2001316

3. COVID-19 Weekly Epidemiological Update. (2021). Accessed: February 16, 2021: https://www.who.int/publications/m/item/weekly-epidemiological-update---16-february-2021.

4. Rudberg AS, Havervall S, Månberg A, et al.: SARS-CoV-2 exposure, symptoms and seroprevalence in healthcare workers in Sweden. Nat Commun. 2020, 11:5064. 10.1038/s41467-020-18848-0

5. Alsuwaidi AR, Al Hosani FI, Al Memari S, et al.: Seroprevalence of COVID-19 infection in the Emirate of Abu Dhabi, United Arab Emirates: a population-based cross-sectional study. Int J Epidemiol. 2021, 50:1077-90. 10.1093/ije/dyab077

6. Murhekar MV, Bhatnagar T, Selvaraju S, et al.: Prevalence of SARS-CoV-2 infection in India: findings from the national serosurvey, May-June 2020. Indian J Med Res. 2020, 152:48-60. 10.4103/ijmr.IJMR_3290_20

7. Selvaraju S, Kumar MS, Thangaraj JW, et al.: Population-based serosurvey for severe acute respiratory syndrome coronavirus 2 transmission, Chennai, India. Emerg Infect Dis. 2021, 27:586-9. 10.3201/eid2702.203938

8. Prakash O, Solanki B, Sheth J, et al.: Serosurveillance among COVID-19 Cases in Ahmedabad Using SARSCOV2 IgG antibodies. Indian J Comm Health. 2021, 33:351-6. 10.47203/IJCH.2021.v33i02.022

9. Hajian-Tilaki K: Sample size estimation in epidemiologic studies . Caspian J Intern Med. 2011, 2:289-98.

10. Arya R, Antonisamy B, Kumar S: Sample size estimation in prevalence studies . Indian J Pediatr. 2012, 79:1482-8. 10.1007/s12098-012-0763-3

11. Andaman and Nicobar Islands India's first to be 'Covid-free' . (2021). Accessed: February 3, 2021: https://timesofindia.indiatimes.com/india/andaman-and-nicobar-islands-indias-first-to-be-covidfree/articleshow/80657....

12. Clinical guidance for management of adult Covid 19 patients. (2021). Accessed: July 31, 2021: http://:https://covid.aiims.edu/clinical-guidance-for-management-of-adult-covid-19-patients/.

13. Sharma N, Sharma P, Basu S, et al.: The seroprevalence of severe acute respiratory syndrome coronavirus 2 in Delhi, India: a repeated population-based seroepidemiological study [PREPRINT]. Trans R Soc Trop Med Hyg. 2021, 10.1093/trstmh/trab109

14. Kshatri JS, Bhattacharya D, Kanungo S, et al.: Findings fromserological surveys (in August 2020) to assess the exposure of adult population to SARS Cov-2 infection in three cities of Odisha, India. medRxiv. 2020, 1:10-1101. 10.1101/2020.10.11.20210807v1

15. Murhekar MV, Bhatnagar T, Selvaraju S, Kumar VS, Vivian TJW, Shah N: SARS-CoV-2antibody seroprevalence in India, August-September 2020: findings from the second nationwide household serosurvey. Lancet Glob Health. 2021, 9:257-66. 10.1016\%2FS2214-109X(20)30544-1

16. Nikolai LA, Meyer CG, Kremsner PG, Velavan TP: Asymptomatic SARS Coronavirus 2 infection: invisible yet invincible. Int J Infect Dis. 2020, 100:112-6. 10.1016/j.ijid.2020.08.076

17. Sekine T, Perez-Potti A, Rivera-Ballesteros O, et al.: Robust $\mathrm{T}$ cell immunity in convalescent individuals with asymptomatic or mild COVID-19. Cell. 2020, 183:158-68.e14. 10.1016/j.cell.2020.08.017

18. Lumley SF, O'Donnell D, Stoesser NE, et al.: Antibody status and incidence of SARS-CoV-2 infection in health care workers. N Engl J Med. 2021, 384:533-40. 10.1056/NEJMoa2034545 


\section{Cureus}

19. The ageing immune system and COVID-19. (2020). Accessed: February 4, 2022: https://www.immunology.org/sites/default/files/BSI_Ageing_COVID-19_Report_Nov2020_FINAL.pdf.

20. Wang X, Guo X, Xin Q, et al.: Neutralizing antibody responses to severe acute respiratory syndrome coronavirus 2 in coronavirus disease 2019 inpatients and convalescent patients. Clin Infect Dis. 2020, 71:2688-94. 10.1093/cid/ciaa721

21. Cervia C, Nilsson J, Zurbuchen Y, et al.: Systemic and mucosal antibody responses specific to SARS-CoV-2 during mild versus severe COVID-19. J Allergy Clin Immunol. 2021, 147:545-57.e9. 10.1016/j.jaci.2020.10.040

22. Ni L, Ye F, Cheng ML, et al.: Detection of SARS-CoV-2-specific humoral and cellular immunity in COVID-19 convalescent individuals. Immunity. 2020, 52:971-7.e3. 10.1016/j.immuni.2020.04.023

23. Rodeles LM, Peverengo LM, Benítez R, et al.: Seroprevalence of anti-SARS-CoV-2 IgG in asymptomatic and pauci-symptomatic people over a 5 month survey in Argentina. Rev Panam Salud Publica. 2021, 45 :e66. 10.26633/RPSP.2021.66

24. Favara DM, McAdam K, Cooke A, et al.: SARS-CoV-2 infection and antibody seroprevalence among UK healthcare professionals working with cancer patients during the first wave of the COVID-19 pandemic. Clin Oncol (R Coll Radiol). 2021, 33:667-75. 10.1016/j.clon.2021.04.005

25. Transmission of SARS-CoV- 2: implications for infection prevention precautions: scientific brief . (2020), Accessed: July 9, 2020: http:////apps.who.int/iris/handle/10665/333114.

26. World Health Organization: Infection Prevention and Control of Epidemic-and Pandemic-prone Acute Respiratory Infections in Health Care. World Health Organization, Geneva; 2014.

27. Garcia-Beltran WF, Lam EC, Astudillo MG, et al.: COVID-19-neutralizing antibodies predict disease severity and survival. Cell. 2021, 184:476-88.e11. 10.1016/j.cell.2020.12.015

28. Legros V, Denolly S, Vogrig M, et al.: A longitudinal study of SARS-CoV-2-infected patients reveals a high correlation between neutralizing antibodies and COVID-19 severity. Cell Mol Immunol. 2021, 18:318-27. 10.1038/s41423-020-00588-2 